\title{
Hodgkin's lymphoma emerging radiation treatment techniques: trade-offs between late radio-induced toxicities and secondary malignant neoplasms
}

Laura Cella ${ }^{1,2}$, Manuel Conson ${ }^{2}$, Maria Cristina Pressello ${ }^{3}$, Silvia Molinellii, Uwe Schneider ${ }^{5}$, Vittorio Donato ${ }^{6}$, Roberto Orecchia ${ }^{7}$, Marco Salvatore ${ }^{2}$ and Roberto Pacelli, ${ }^{1,2 *}$

\begin{abstract}
Background: Purpose of this study is to explore the trade-offs between radio-induced toxicities and second malignant neoplasm (SMN) induction risk of different emerging radiotherapy techniques for Hodgkin's lymphoma $(\mathrm{HL})$ through a comprehensive dosimetric analysis on a representative clinical model.

Methods: Three different planning target volume $\left(P T V_{i}\right)$ scenarios of a female patient with supradiaphragmatic $\mathrm{HL}$ were used as models for the purpose of this study. Five treatment radiation techniques were simulated: an anterior-posterior parallel-opposed (AP-PA), a forward intensity modulated (FIMRT), an inverse intensity modulated (IMRT), a Tomotherapy (TOMO), a proton (PRO) technique. A radiation dose of 30 Gy or CGE was prescribed. Dose-volume histograms of PTVs and organs-at-risk (OARs) were calculated and related to available dose-volume constraints. SMN risk for breasts, thyroid, and lungs was estimated through the Organ Equivalent Dose model considering cell repopulation and inhomogeneous organ doses.

Results: With similar level of PTV ${ }_{i}$ coverage, IMRT, TOMO and PRO plans generally reduced the OARs' dose and accordingly the related radio-induced toxicities. However, only TOMO and PRO plans were compliant with all constraints in all scenarios. For the IMRT and TOMO plans an increased risk of development of breast, and lung SMN compared with AP-PA and FIMRT techniques was estimated. Only PRO plans seemed to reduce the risk of predicted SMN compared with AP-PA technique.
\end{abstract}

Conclusions: Our model-based study supports the use of advanced RT techniques to successfully spare OARs and to reduce the risk of radio-induced toxicities in $\mathrm{HL}$ patients. However, the estimated increase of SMNs' risk inherent to TOMO and IMRT techniques should be carefully considered in the evaluation of a risk-adapted therapeutic strategy.

Keywords: Hodgkin's lymphoma, Emerging radiotherapy techniques, Radio-induced toxicity, Second malignant neoplasm

\footnotetext{
* Correspondence: pacerto@yahoo.com

${ }^{1}$ Institute of Biostructures and Bioimaging, National Council of Research

(CNR), Napoli, Italy

${ }^{2}$ Department of Diagnostic Imaging and Radiation Oncology, University

"Federico II" of Napoli, Napoli, Italy

Full list of author information is available at the end of the article
} 


\section{Background}

In the past decades, treatment improvements have made Hodgkin's lymphoma (HL) one of the most curable malignancies. However, due to the low patients mean age, the combined use of potentially harmful therapeutic agents and the efficiency of the therapy that allows a high cure rate with a long life span expectation, late effects of HL treatment represent an important and considerable threat for surviving patients. Indeed, the older series of successfully treated long term surviving patients showed a high rate of late side effects of therapy including iatrogenic lung, heart and thyroid diseases [1-3].

Technological advances in HL radiation therapy (RT) [4-11] by high conformal treatments potentially increase control over organs-at-risk (OAR) dose distribution. Dose-volume histogram (DVH) predictors in HL patients have been reported for late side effects such as radiation pneumonitis [12], hypothyroidism [13], and cardiovascular diseases [14,15] supporting the planning optimization procedures so as to limit OAR complication risks.

However, considering the low mean age, the high cure rate, and the consequent long survival expectation of HL patients, caution must be taken in the application of modern techniques such as intensity modulated radiotherapy or Tomotherapy because of the greater volume of normal tissue receiving low-to-moderate radiation doses and their inherent risk of second malignant neoplasms (SMNs) that may be significantly higher compared with 3D conformal radiotherapy [16]. Moreover, the impact on SMN incidence from particle therapy producing secondary neutrons causes some concern [17]. Structures with a high potential for the development of second malignancies, such as lung, thyroid and breast, must be considered.

Predicting SMN risk from these newer and sophisticated RT delivery techniques is complicated by their having been only recently introduced and by the consequent absence of epidemiological data [18]. As an alternative, biologicallybased mathematical models can be used to estimate the risk of SMNs related to a given RT technique using organ dose distribution through dose-volume histograms [19-23]. These models allow to compare dose distributions with regard to the estimated risk of SMNs in the irradiated organs as a function of point dose in the radiotherapy dose range also including fractionation effects.

The aim of this study is to analyze normal tissue sparing capability of different RT techniques for one representative supradiaphragmatic HL model case, in particular to explore the trade-offs between radio-induced toxicities and SMNs induction risk. For this purpose, we have conceived three different size planning target volumes (PTVs), each with different involvement of OARs such as heart, thyroid, breasts and lungs. We have simulated RT plans using five different delivery techniques. DVHs were then used to predict the impact of the different analysed RT techniques on late side effects and on SMN induction risk estimated through the Organ Equivalent Dose (OED) model considering cell repopulation and inhomogeneous organ doses [20].

\section{Methods}

Planning CT-scan of a female patient with supradiaphragmatic HL in standard supine position with 5 -mm slices acquisition was considered. Different involved field clinical target volume $\left(\mathrm{CTV}_{\mathrm{i}}\right)$ size scenarios were generated: small $\left(\mathrm{CTV}_{1}\right)$, medium $\left(\mathrm{CTV}_{2}\right)$, and large $\left(\mathrm{CTV}_{3}\right)$. The $\mathrm{CTV}_{1}$ included the upper mediastinal and left supraclavear nodal sites; the $\mathrm{CTV}_{2}$ included $\mathrm{CTV}_{1}$ plus bilateral lung hylus; the $\mathrm{CTV}_{3}$ included the whole mediastinum, the bilateral lung hylus, and bilateral supraclavear nodal sites. The nodal sites were delineated as described elsewhere [24]. Planning target volumes $\left(\mathrm{PTV}_{\mathrm{i}}\right)$ included $\mathrm{CTV}_{\mathrm{i}}$ plus a $10 \mathrm{~mm}$ margin (Figure 1). The following OARs were contoured: bilateral lungs, whole heart, cardiac chambers, pericardium, thyroid and breasts. For cardiac structures delineation, the heart atlas [25] was applied while breasts were defined as described by Weber et al. [26].

\section{Radiotherapy techniques}

Five treatment plans were generated on purpose for each $\mathrm{PTV}_{\mathrm{i}}$ : a conventional anterior-posterior parallel-opposed (AP-PA) plan, a forward intensity modulated plan (FIMRT), an inverse intensity modulated plan (IMRT), a Tomotherapy plan (TOMO), and a proton plan (PRO). A total dose of 30 Gy or cobalt gray equivalent (CGE) in 20 daily fractions of 1.5 Gy was planned. All treatment plans were optimized to ensure $95 \%$ of the prescription dose delivered at least to $95 \%$ of the PTV with a maximum dose less than $115 \%$.

\section{AP-PA}

Conventional AP-PA plans were simulated using photon beams from a linac equipped with 40 pairs of multileaf collimator (MLC). Treatment planning was performed by a 3-D planning system (XiO, Elekta-CMS) and a convolution dose calculation algorithm was applied.

\section{FIMRT}

A step-by-step iterative process inherent to forward planning was used manually adding two or more MLC shaped subfields with the same AP-PA isocenter and gantry position. Treatment plans were generated with XIO planning system; the MLC positions and beam weightings were optimized by forward planning based on the 3D dose distribution as well as on DVHs.

\section{IMRT}

Seven field IMRT treatments were planned with Pinnacle3 TPS (Philips) using Direct Machine Parameter Optimization and Cone Convolution algorithm (CCA) for 


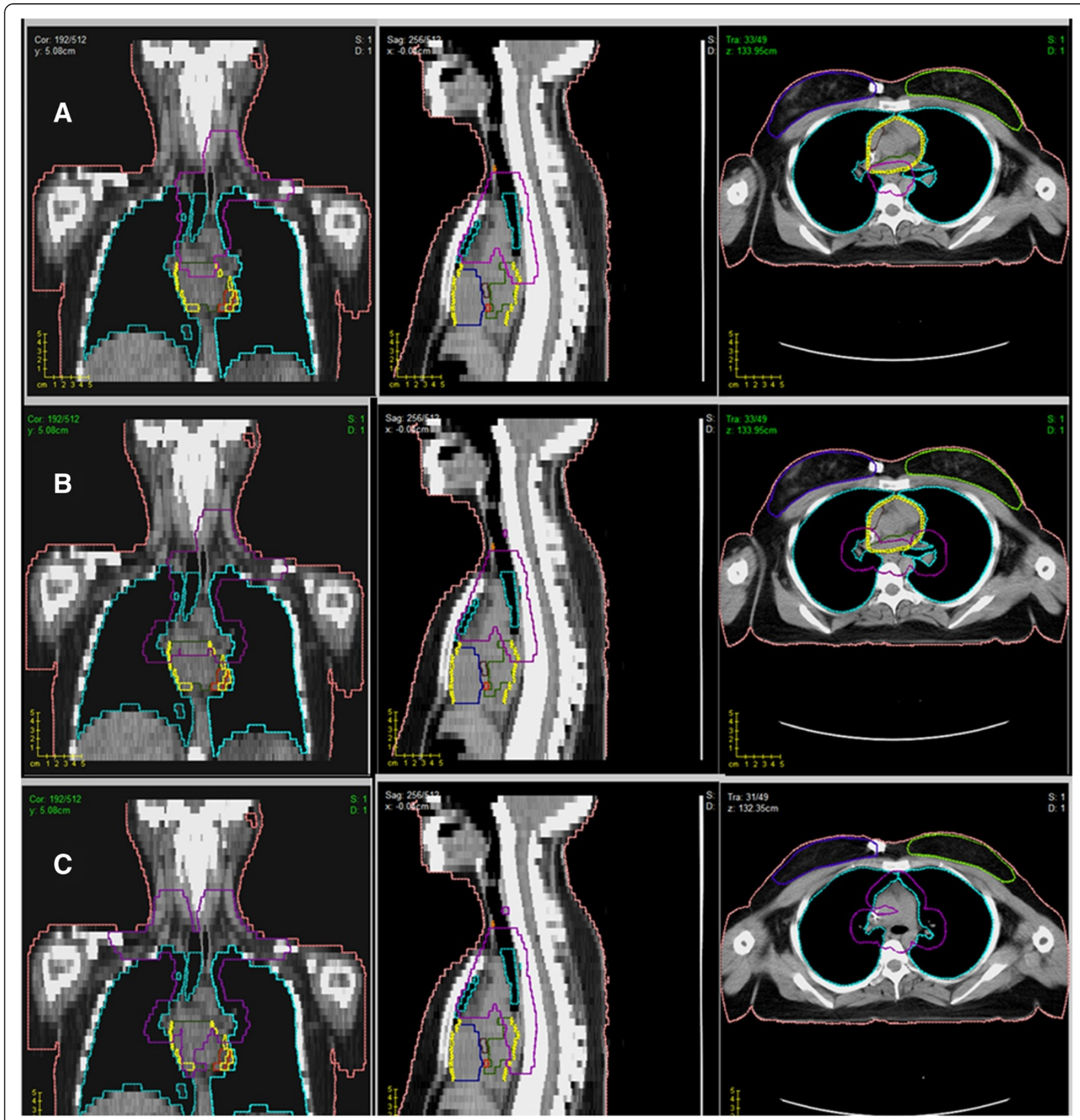

Figure 1 Three different planning target volume size scenarios: A) small $\left(\right.$ PTV $\left._{1}\right)$, B) medium $\left(\right.$ PTV $\left._{2}\right)$, C) large $\left(\right.$ PTV $\left._{3}\right)$. PTVi are outlined in purple.

dose calculation and a Siemens Artiste linac, with a step-and-shoot technique performed with the 160 leaves collimator.

\section{TOMO}

Tomotherapy treatments were planned with Tomotherapy Planning Station (Accuray) with gradient descent optimization algorithm, establishing a 2.5 field width, 0,287 pitch value and a starting Modulation Factor
(MF) of 4 and an actual MF of 3.9, 3.6 and 2.7 for PTV , $_{1}$ $\mathrm{PTV}_{2}$, and $\mathrm{PTV}_{3}$ plans, respectively. The dose distributions were calculated with CCA. Delivery was performed with fifty-one fields for each gantry rotation and beam modulation carried out with a 64 leaves binary collimator.

\section{PRO}

Proton plans were generated with the Syngo RT Planning Station (Siemens VB-10), using an active scanning dose 
delivery system (Centro Nazionale di Adroterapia Oncologica Foundation). Proton energies from 62 to $180 \mathrm{MeV} / \mathrm{u}$ were used with a nominal $10 \mathrm{~mm}$ Full Width Half Maximum pencil beam focus and a beam intensity of $2^{*} 10^{9}$ particles per spill. A scanning step of $3 \mathrm{~mm}$ was fixed for the transversal directions and a $2 \mathrm{~mm}$ energy step was selected for Spread Out Bragg Peak generation. For $\mathrm{PTV}_{1}$ and $\mathrm{PTV}_{2}$, an AP-PA configuration was defined, while two couples of parallel-opposed beams, centered on the PTV, were applied for $\mathrm{PTV}_{3}$. A $4 \mathrm{~cm}$ range shifter was introduced for the anterior beam directions to achieve the minimum proton energy required. A fixed RBE value of 1.1 was used.

IMRT, TOMO and PRO plans were optimized using constraints on the OARs and priority weightings published by Weber et al. [26].

All treatment characteristics including the delivered monitor units and the number of used protons are summarized in Table 1. The contribution from the scattered neutrons was considered and the dose distribution corrected using the data from d'Errico et al. [27] for

Table 1 Treatment techniques characteristics

\begin{tabular}{|c|c|c|c|c|}
\hline${ }_{\text {Technique- } P T V_{i}}$ & $\begin{array}{c}\text { Fields or } \\
\text { Subfields } \\
\text { number }\end{array}$ & $\begin{array}{c}\text { Energy } \\
(\mathrm{MeV})\end{array}$ & $\begin{array}{l}\text { Total MUs or } \\
\text { protons per Gy }\end{array}$ & $\begin{array}{c}\text { Neutron } \\
\text { equivalent } \\
\text { dose (Sv) }\end{array}$ \\
\hline AP-PA- PTV 1 & 2 & 6 & 5040 & 0 \\
\hline AP-PA- PTV 2 & 2 & 6 & 5038 & 0 \\
\hline AP-PA- PTV 3 & 2 & 6 & 4157 & 0 \\
\hline \multirow[t]{2}{*}{ FIMRT- PTV } & 1 & 6 & 1427 & 0 \\
\hline & 3 & 15 & 2070 & 0.020 \\
\hline \multirow[t]{2}{*}{ FIMRT- PTV 2} & 1 & 6 & 1415 & 0 \\
\hline & 3 & 15 & 2040 & 0.020 \\
\hline \multirow[t]{2}{*}{ FIMRT-PTV $_{3}$} & 3 & 6 & 1714 & 0 \\
\hline & 3 & 15 & 2277 & 0.020 \\
\hline IMRT- PTV 1 & 7 & 6 & 8020 & 0 \\
\hline IMRT- PTV 2 & 7 & 6 & 9500 & 0 \\
\hline IMRT- PTV 3 & 7 & 6 & 14000 & 0 \\
\hline TOMO- PTV 1 & & 6 & 10042 & 0 \\
\hline TOMO- PTV 2 & & 6 & 9661 & 0 \\
\hline $\mathrm{TOMO}^{-\mathrm{PTV}_{3}}$ & & 6 & 8232 & 0 \\
\hline \multirow[t]{2}{*}{ PROTONS- PTV } & 1 & $88-170$ & $5.43^{*} 10^{10}$ & 0.16 \\
\hline & 1 & 62-162 & $4.52^{*} 10^{10}$ & \\
\hline \multirow[t]{2}{*}{ PROTONS- PTV 2} & 1 & 87-171 & $6.20^{*} 10^{10}$ & 0.19 \\
\hline & 1 & 62-162 & $4.52^{*} 10^{10}$ & \\
\hline \multirow[t]{4}{*}{ PROTONS- PTV 3} & 1 & 88-180 & $3.52^{*} 10^{10}$ & 0.23 \\
\hline & 1 & $88-173$ & $3.92^{*} 10^{10}$ & \\
\hline & 1 & 62-162 & $3.42^{*} 10^{10}$ & \\
\hline & 1 & 62-166 & $3.34^{*} 10^{10}$ & \\
\hline
\end{tabular}

photons, and the data from Schneider et al. [28] for spotscanned protons, for neutron equivalent dose estimation. The following neutron equivalent dose in Sv per applied MUs and per treatment protons per Gy were used: $\mathrm{H}^{N, 6 M V}=0, \mathrm{H}^{N, 15 M V}=1 \times 10^{-5}, \mathrm{H}^{N, \text { protons }}=6 \times 10^{-14}$. The out-of-axis neutron dose contribution was neglected since the OARs we considered for plan evaluation were included in the primary dose distribution. However, for organs far from the target volume which were not considered in the present study the out-of-field neutron contribution can be important.

\section{Plan evaluation}

For each RT technique and for each PTV scenario specific organ dose-volume metrics and dose parameters were calculated from DVHs and related to available predictors for radio-induced toxicities:

Whole Heart: V25 <10\% [15]; endpoint: long-term cardiac mortality;

Cardiac Chambers: Left Atrium V25<63\%; Left Ventricle V30<25\%; Right Ventricle V30 $\leq 65 \%$ [14]; endpoint: asymptomatic heart valvular dysfunction;

Pericardium: V30 $\leq 46 \%$; mean dose $\leq 26$ Gy [15]; endpoint: pericarditis;

Lungs: V20 $\leq 33.5 \%$; mean dose $\leq 13.5$ Gy [12]; endpoint: symptomatic radiation pneumonitis;

Thyroid: V30 $\leq 62 \%$ [13]; endpoint: clinical or subclinical hypothyroidism.

Where VX is the percentage of organ volume exceeding X Gy.

Of note, the threshold metrics for cardiac chambers, lungs, and thyroid were specifically extrapolated from HL patients' cohorts.

The DVHs were also utilized to estimate the SMN risk for breasts, lungs and thyroid through the application of the OED concept using specific organ model-parameters. Accordingly, the risk ratio (RR) for a RT plan $i$ relative to another plan $j$ with respect to cancer induction in one organ is equivalent to the OED ratio [20]:

$$
\mathrm{RR}_{i j}=\frac{O E D\left(\operatorname{plan}_{i}\right)}{\mathrm{OED}\left(\operatorname{plan}_{j}\right)}
$$

The AP-PA plan was used as reference for $R R_{i j}$ calculation.

The OED can be determined on the basis of an organ specific dose-response relationship for radiation induced cancer ("risk equivalent dose", RED) and DVH. The RED for carcinoma induction is given by a mechanistic model 
accounting for cell killing and fractionation effects [19]. Briefly, for a given organ and a given plan:

$$
O E D=\frac{1}{V_{T}} \sum_{i} V\left(D_{i}\right) R E D\left(D_{i}\right)
$$

where $V_{T}$ is the total organ volume and the sum is taken over all DVH bins, and

$$
R E D(D)=\frac{e^{-\alpha^{\prime} D}}{\alpha^{\prime} R}\left(1-2 R+R^{2} e^{\alpha^{\prime} D}-(1-R)^{2} e^{-\frac{\alpha^{\prime} R}{1-R} D}\right)
$$

where $\alpha^{\prime}=\alpha+\beta d$, with $\alpha$ and $\beta$ denoting the linearquadratic model parameters for the organ of interest and $d$ the dose fraction, $D$ the total dose, and $R$ the repopulation/repair parameter. The dose-response model is robust with variations in $\alpha / \beta$ [20] and an $\alpha / \beta=3$ Gy was used for all calculations. The risk for secondary breast, thyroid and lung cancers were estimated with parameter values $\alpha=0.067 ; 0.0318 ; 0.042 \mathrm{~Gy}^{-1}$ and $R=0.62$; 0.0 ; 0.83 respectively [20]. Using RED (D) given by eq. (3), the risk-volume histograms (RVHs) for breast, thyroid and lung cancers were calculated.

\section{Results}

Target sizes were $\mathrm{PTV}_{1}=497.0 \mathrm{~cm}^{3}, \mathrm{PTV}_{2}=626.7 \mathrm{~cm}^{3}$, and $\mathrm{PTV}_{3}=837.4 \mathrm{~cm}^{3}$. All RT techniques succeeded in obtaining the requested $\mathrm{PTV}_{\mathrm{i}}$ dose coverage independently of PTV size. Comparative DVHs for the different PTV $_{i}$ and for all techniques are shown in Additional file 1. PTV coverage was optimal with both TOMO and PRO plans.

\section{Radiation dose to OARs}

In Table 2 are reported the DVH parameters for the different RT plans and for each $\mathrm{PTV}_{\mathrm{i}}$. With regard to $\mathrm{PTV}_{1}$ and $\mathrm{PTV}_{2}$ scenarios, DVH analysis (Additional file $2 \mathrm{a}$ and $2 \mathrm{~b}$ ) shows that all the different techniques respected the considered constraints with the exception of the whole-heart V25 for which only the TOMO and PRO plans were able to reduce it under $10 \%$. Regarding the $\mathrm{PTV}_{3}$ the AP-PA, FIMRT, IMRT plans violate the dose-volume limits for the whole-heart and for the left atrium. In addition, the AP-PA plan exceeds the $62 \%$ volume for thyroid V30 and the IMRT plan shows a mean lung dose just equal to 13.5 Gy limit (Additional file 2c).

In general, the PRO and TOMO plans provided the lowest parameter values for all the considered OARs and spared them better than IMRT plan.

\section{SMNs relative risk}

The estimated OED values for breasts, lungs and thyroid for all RT techniques are listed in Table 3. In Figure 2 comparative RVHs for the above OARs are shown. In breasts and lungs, the PRO plan provided the lowest
Table 2 Organ dose-volume metrics and dose parameters

\begin{tabular}{|c|c|c|c|c|c|c|}
\hline & & $A P-P A$ & FIMRT & IMRT & TOMO & PRO \\
\hline \multicolumn{7}{|l|}{ PTV $_{1}$} \\
\hline Heart & V25 & $22.5^{*}$ & $21.0^{*}$ & $11.8^{*}$ & 4.5 & 3.6 \\
\hline Left Atrium & V25 & 42.2 & 39.2 & 35.0 & 25.8 & 24.5 \\
\hline Left ventricle & V30 & 0.1 & 0.1 & 0.1 & 0.1 & 0.1 \\
\hline Right Ventricle & V30 & 7.6 & 2.4 & 0.1 & 0.1 & 0.1 \\
\hline \multirow[t]{2}{*}{ Pericardium } & V30 & 6.2 & 2.5 & 3.9 & 3.0 & 2.1 \\
\hline & mean dose & 10.0 & 9.2 & 6.4 & 7.7 & 4.3 \\
\hline Thyroid & V30 & 49.0 & 48 & 41.4 & 16.9 & 28.5 \\
\hline \multirow[t]{2}{*}{ Lungs } & V20 & 12.1 & 11 & 15 & 10.1 & 8.2 \\
\hline & mean dose & 5.3 & 4.9 & 7.8 & 8.1 & 3.3 \\
\hline \multicolumn{7}{|l|}{ PTV $_{2}$} \\
\hline Heart & V25 & $27.7^{*}$ & $29.9^{*}$ & $12.3^{*}$ & 5.0 & 6.7 \\
\hline Left Atrium & V25 & 41.8 & 45.0 & 41.5 & 27.2 & 30.4 \\
\hline Left ventricle & V30 & 9.2 & 7.5 & 0 & 0.1 & 0.3 \\
\hline Right Ventricle & V30 & 9.7 & 6.5 & 0 & 0 & 0 \\
\hline \multirow[t]{2}{*}{ Pericardium } & V30 & 14.9 & 11.5 & 9.2 & 5.5 & 2.9 \\
\hline & mean dose & 11.8 & 12.5 & 10.7 & 7.8 & 7.5 \\
\hline Thyroid & V30 & 49.3 & 42.1 & 27.8 & 16 & 18.6 \\
\hline \multirow[t]{2}{*}{ Lungs } & V20 & 24.7 & 23.6 & 20.5 & 16.5 & 14.4 \\
\hline & mean dose & 9.6 & 9.2 & 10.5 & 9.7 & 6.4 \\
\hline \multicolumn{7}{|l|}{$\mathrm{PTV}_{3}$} \\
\hline Heart & V25 & $60.5^{*}$ & $67.5^{*}$ & $22.0^{*}$ & 8.7 & 7.3 \\
\hline Left Atrium & V25 & $98.3^{*}$ & $99.3^{*}$ & $73.0^{*}$ & 49.4 & 43.0 \\
\hline Left ventricle & V30 & 12.0 & 13.0 & 2.5 & 0.1 & 0 \\
\hline Right Ventricle & V30 & 49.2 & 47.0 & 0 & 0 & 0 \\
\hline \multirow[t]{2}{*}{ Pericardium } & V30 & 31.3 & 42.0 & 20.6 & 13.0 & 3.2 \\
\hline & mean dose & 22.0 & 23.8 & 17.2 & 14.6 & 10.2 \\
\hline Thyroid & V30 & $93.9^{*}$ & 24.0 & 60 & 45.0 & 7.0 \\
\hline \multirow[t]{2}{*}{ Lungs } & V20 & 30.7 & 28.8 & 27.0 & 23.0 & 14.5 \\
\hline & mean dose & 11.9 & 11.1 & $13.5^{*}$ & 12.6 & 6.5 \\
\hline
\end{tabular}
for the different RT plans and for each PTV

* not compliant with constraint

$V X$ is the percentage of organ volume exceeding X Gy.

risk-volume curve whereas the highest curves were provided by IMRT and TOMO plans.

RR values for FIMRT, IMRT, TOMO and PRO plans relative to the AP-PA plan with respect to SMN induction in breasts, lungs and thyroid are plotted in Figure 3.

Regarding breast cancer induction, in the $\mathrm{PTV}_{1}$ case, the IMRT and TOMO plans exhibit OED values of 2.44 and 2.13, respectively, which result in a RR for SMN induction that is 2.2- and 2.0-fold the risk of a AP-PA plan (Figure 3A). The IMRT and TOMO plans' RR for breast cancer induction exhibit a small reduction when the PTV size increases; nonetheless we observe an approximately 2 -fold increase compared with AP-PA or 
Table 3 Estimated Organ Equivalent Dose (OED) for the different RT plans and for each PTV $V_{i}$

\begin{tabular}{llll}
\hline & $\begin{array}{c}P T V_{1} \\
\text { OED }\end{array}$ & $\begin{array}{c}\boldsymbol{P T V}_{2} \\
\text { OED }\end{array}$ & $\begin{array}{c}\boldsymbol{P T V}_{\mathbf{3}} \\
\text { OED }\end{array}$ \\
\hline Breasts & & & \\
AP-PA & 1.09 & 1.41 & 2.25 \\
FIMRT & 0.94 & 1.92 & 2.52 \\
IMRT & 2.44 & 3.00 & 3.91 \\
TOMO & 2.13 & 2.71 & 4.02 \\
PRO & 0,35 & 0,94 & 1.14 \\
Lungs & & & \\
AP-PA & 2.82 & 4.48 & 5.58 \\
FIMRT & 2.64 & 4.38 & 5.34 \\
IMRT & 3.77 & 4.98 & 6.25 \\
TOMO & 4.60 & 5.35 & 6.76 \\
PRO & 1.67 & 2.98 & 3.30 \\
Thyroid & & & \\
AP-PA & 6.35 & 6.31 & 6.98 \\
FIMRT & 6.26 & 6.17 & 7.25 \\
IMRT & 7.69 & 7.71 & 7.12 \\
TOMO & 7.49 & 6.30 & 7.20 \\
PRO & 7.35 & 6.50 & 7.34 \\
\hline
\end{tabular}

FIMRT techniques. On the contrary, in all PTV scenarios, PRO plan gives an OED in the range of 0.35-1.14 and accordingly a RR induction compared with conventional plan in the range of 0.3-0.7. When IMRT and TOMO were compared to PRO plan, we observed an increase in $\mathrm{RR}$ values for the breast by a factor ranging from a minimum of 3 (TOMO in $\mathrm{PTV}_{2}$ ) to a maximum of 7 (IMRT in PTV $)_{1}$ ).

For lung cancer induction (Figure 3B), we observed the same behavior for the TOMO and IMRT techniques increasing RR values by a factor 1.1-1.6 compared with the conventional plan, while with PRO plans we observed a RR reduction. Conversely, for thyroid (Figure $3 \mathrm{C}$ ) the $\mathrm{RR}$ values are close to 1 for all the techniques and all target sizes except for IMRT in PTV and $\mathrm{PTV}_{2}$ cases and for TOMO in the $\mathrm{PTV}_{1}$ case in which the RR is 1.2.

\section{Discussion}

Since the implementation in HL therapy of extended field irradiation, the high cure rate was offset by late side effects and development of SMNs in a relevant fraction of patients [1]. The progresses in imaging and the better knowledge of the disease biology, with consequent better prognostic stratification of patients, have allowed a decrease in the therapeutic load consisting in a progressive reduction of chemotherapy cycles, radiation dose and treated volume in most patients [29]. However, the risk of late iatrogenic effects remains remarkable. The radiation delivery techniques can heavily condition the distribution of the dose in tissues and alter the toxicity profile of a treatment. Involved-field IMRT has shown excellent target coverage and amelioration of side effects in a clinical study by $\mathrm{Lu}$ et al. [7]. Volumetric modulated arc therapy has been shown to significantly reduce hearth dose in HL patients affected with cardiovascular disease [30] and to perform better than IMRT in sparing the OARs when using involved nodal RT $[10,26]$. With the same purpose Tomotherapy has been recently proposed for the treatment of HL [8]. Recent preliminary studies of proton beam therapy for mediastinal HL have been reported [4]. The OARs toxicities and development of second breast neoplasms would be expected to be reduced by the use of particle therapy. However, while it is epidemiologically reasonable to expect that a dose reduction is associated with a reduced risk of late effects, an improvement in SMN risk due to dose reduction is not yet clearly established.

Our study aims at analyzing 5 different radiation delivery techniques in three different hypothetical scenarios of supradiaphragmatic HL through a comprehensive dosimetric study. The main endpoint was to investigate, for each single technique, the balance between the predicted OARs injuries and the predicted development of SMNs, with the same target optimal dose coverage. The advantage of IMRT for heart and left ventricle sparing as well as its disadvantages in the low dose region, in particular for breasts, have been already reported in the literature [9]. However, in our study the above advantages and disadvantages were quantified and extended to other state-of-the-art techniques.

As surrogate indicators of OARs morbidities, some of the constraints recently suggested by the literature were used. We chose constraints predictive of feared radioinduced injuries commonly described in patients treated with sequential chemo-radiotherapy for $\mathrm{HL}$ such as hypothyroidism [13], asymptomatic cardiac valvular dysfunction [14] and radiation pneumonitis [12] and specifically extrapolated from HL patients' cohorts, together with some other more general constraints suggested by QUANTEC reviews [15].

Lacking epidemiological data relative to the recent RT delivery techniques, estimation of the risk of SMN for breasts, lungs, and thyroid based on mathematical models $[19,20]$ was used. Many uncertainties are involved in modelling the underlying biology of radiation induced-cancer. Nevertheless, these models may be reliably used to predict the impact on SMN induction of a given technique relative to another reference technique. To this end, we introduced the concept of risk ratio RR as a parameter for plan evaluation. 


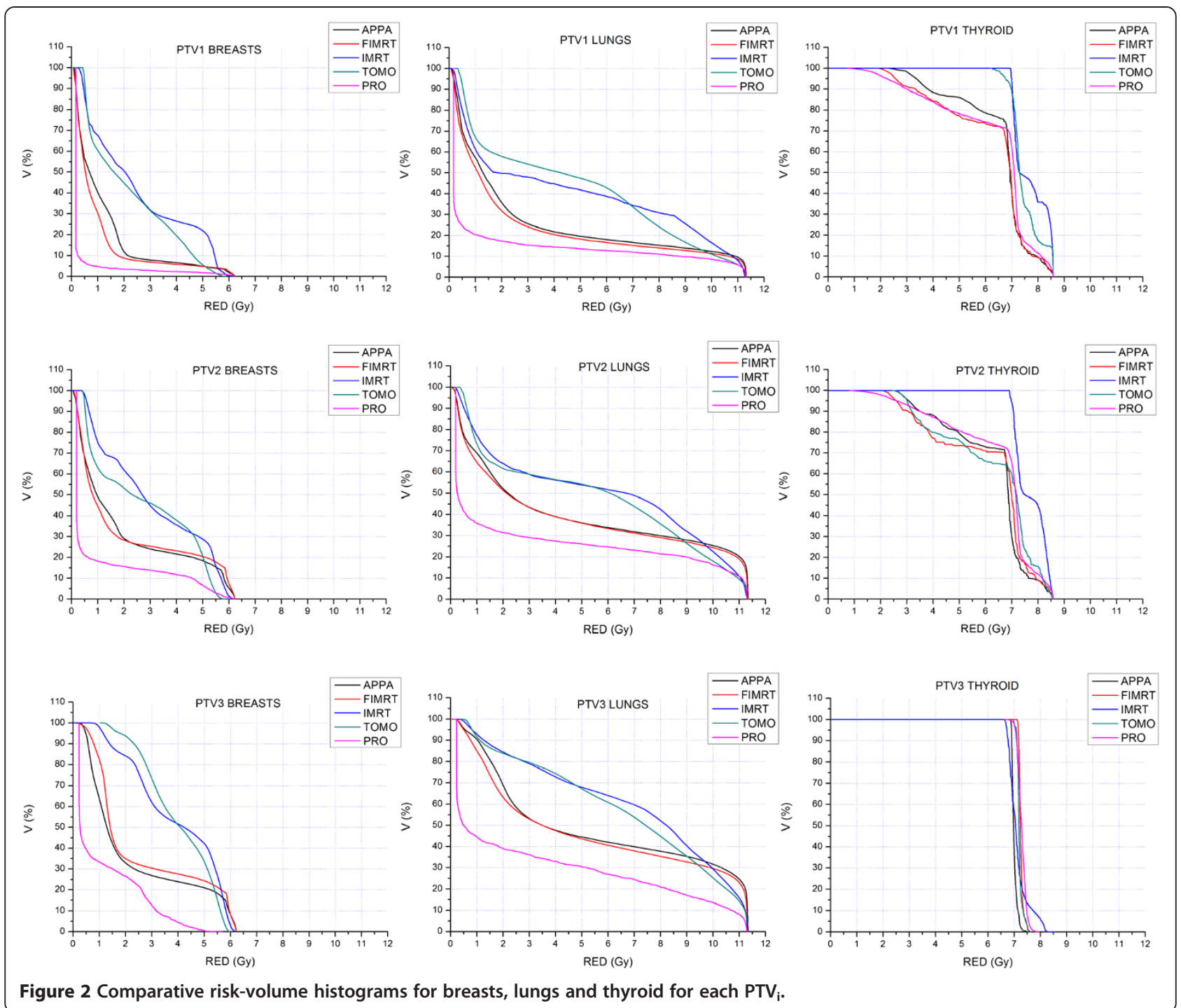

It should be noted that for the quality factor $Q$ (stochastic $\mathrm{RBE}$ ) we take a value of 1.1 for protons. There is a strong energy dependence for the quality factor and a factor of two, as recommended by ICRP 92 [31], would perhaps only be expected at very low energies in the tail of the Bragg peak. The main contribution to the normal tissue integral dose, however, will come from the plateau region of the Bragg curve due to the protons passing through normal tissue to reach the target volume. This portion of the Bragg curve consists predominantly of dose deposited by higher energy protons (much higher than $8 \mathrm{MeV}$ ) for which the NCRP quotes a value of one [32,33]. In the normal tissue distal to the target volume, although the quality factor may be higher, the irradiated volume will be very much smaller and the deposited dose will be lower due to the finite maximum range of protons in the tissue. Therefore, it is safe to assume that the vast majority of normal tissues will be irradiated by protons with a quality factor close to one.
As regards PTV coverage, in the framework of a satisfactory performance of all the above techniques, the optimal coverage was obtained by TOMO and PRO plans.

As far as constraint compliance is concerned, in all PTV scenarios, AP-PA, FIMRT, and IMRT plans exceed the whole-heart-V25 of $10 \%$. This limit, associated with a $<1 \%$ probability of cardiac mortality, is an overly safe risk estimate based on model predictions and consequently the risk may be overestimated [15]. The other constraints were met by all five techniques in $\mathrm{PTV}_{1}$ and $\mathrm{PTV}_{2}$ scenarios. For $\mathrm{PTV}_{3}$, the AP-PA, FIMRT and IMRT also failed to meet left atrium V25 cutoff volume of $63 \%$ which is a significant predictor of mitral and aortic valvular defects. The latter are particularly important for those patients characterized by high cure rates and prolonged survival like HL patients because of their progressive nature and potential contribution to overt cardiac toxicity [14]. Only the AP-PA failed to meet thyroid V30 dose constraint predictive of hypothyroidism. 


\section{A BREASTS}
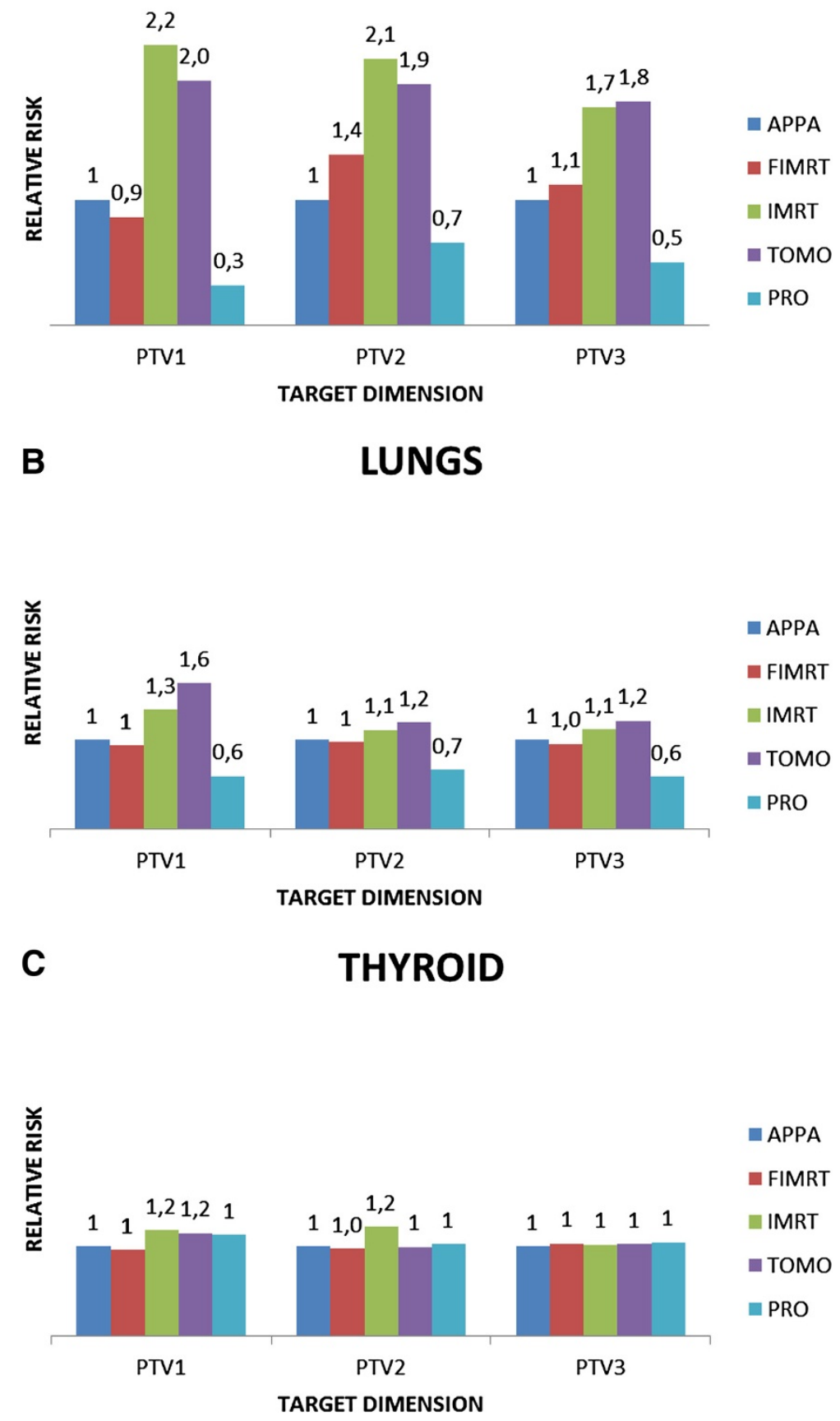

Figure 3 Estimated values of the risk ratio (RR) for FIMRT, IMRT, TOMO and PRO plans relative to the AP-PA plan with respect to SMN induction in breasts, lungs and thyroid.

Remarkably, beyond DVH predictors, TOMO and PRO led to a reduction in the doses to all the OARs compared with the other plans.

Conversely, the estimated risk ratio of SMNs induction for breasts and lungs was significantly increased by IMRT and TOMO in all scenarios though it is lower when the target volume is larger. No relevant risk ratio increase in thyroid cancer was found for any technique. To be noted, theoretically PRO led to a reduction of risk ratio in all cases. Among photon delivery techniques, conventional AP-PA and FIMRT resulted in the lowest estimated risk of SMNs.

This study, exploring the trade-offs between radioinduced toxicities and SMN by planning comparative evaluations, provides informative tools so as to evaluate which 
HL patient potentially deserves a more advanced radiation technique obtaining a real advantage in terms of deterministic and/or stochastic damage prevention. Diverse variables must be considered such as individual patients features, site and size of disease in order to establish strategies capable of performing a risk-adapted radiotherapy.

Let us point to some potential limitations of our proof-of-concept study. First, we considered one single model case not taking into account morphological differences peculiar to each single patient such as heart, lung and breast volumes. We also analyzed three different PTVs that, although paradigmatic, did not cover all possible varieties of HL. Moreover, in SMN estimation the uncertainty linked to neutron RBE for carcinogenesis should be taken into account .

Given the above considerations, our analysis suggests that, as already shown for other tumor sites $[17,34]$, proton therapy could theoretically be the optimal radiation modality in all HL scenarios studied, provided that plan robustness and organ motion are properly managed [35]. However, costs and availability currently limit proton usage. Regarding photon techniques, the choice of the more appropriate treatment should be tailored to the individual case. For instance, for a young male patient with a large tumor or a patient with cardiac co-morbidity both requiring a total dose of $30 \mathrm{~Gy}$, TOMO plan would result extremely advantageous. On the contrary, TOMO could not be equally advantageous for a good prognosis young (25 years) HL bearing female patient requiring a total dose of $20 \mathrm{~Gy}$, which implies a very low risk of late organ injuries. In such a case, radioinduced breast cancer may be of more concern and FIMRT may result more appropriate.

\section{Conclusions}

Our model-based study fosters the use of advanced RT techniques to reduce the dose to OARs and, consequently, the risk of radio-induced toxicities in HL patients. However, in the framework of a modern risk-adapted therapeutic strategy, the estimated increase of SMNs' risk inherent to TOMO and IMRT techniques should be carefully considered.

\section{Additional files}

Additional file 1: Comparative dose-volume histograms for each PTV $_{\mathrm{i}}$ scenario and for all techniques.

Additional file 2: Comparative dose-volume histograms for each organ-at-risk and for all techniques for a) PTV $_{1}$ scenario, b) PTV $_{2}$ scenario, c) PTV3 scenario.

\section{Abbreviations}

AP-PA: Anterior-posterior parallel opposed; CCA: Cone convolution algorithm; CGE: Cobalt Gray equivalent; CTV: Clinical target volume; DVH: Dose-volume histogram; FIMRT: Forward intensity modulated radiation therapy; FWHM: Full width at half maximum; IMRT: Inverse intensity modulated radiation therapy; HL: Hodgkin's lymphoma; MF: Modulation factor; MLC: Multi-leaf collimator;
OAR: Organ-at-risk; OED: Organ equivalent dose; PRO: Proton; PTV: Planning target volume; RED: Risk equivalent dose; RR: Risk ratio; RT: Radiation therapy; RVH: Risk-volume histograms; SMN: Second malignant neoplasm; TOMO: Tomotherapy.

Competing interests

The authors declare no conflict of interest.

\section{Authors' contributions}

LC and RP conceived and designed the study. MC, MS and RP defined target volumes. LC, MC and RP performed conventional technique plans. MCP and VD performed IMRT and TOMO plans. SM and RO performed proton plans. LC and US applied second cancer modeling. LC reviewed and analyzed all dosimetric data. All authors participated in drafting and revising the manuscript. All authors have given their final approval of the manuscript.

\section{Acknowledgements}

The authors gratefully acknowledge Dr. Maria Pia Graziani for the English revision and Professor Guido Cella for useful discussion. This work was partially supported by grants from Italian Ministry for Education, University and Research (MIUR) in the framework of FIRB (RBNE08YFN3 "MERIT").

\section{Author details}

'Institute of Biostructures and Bioimaging, National Council of Research (CNR), Napoli, Italy. ${ }^{2}$ Department of Diagnostic Imaging and Radiation Oncology, University "Federico II" of Napoli, Napoli, Italy. ${ }^{3}$ Department of Health Physics, S. Camillo-Forlanini Hospital, Roma, Italy. ${ }^{4}$ Unit of Medical Physics, Centro Nazionale di Adroterapia Oncologica Foundation, Pavia, Italy. ${ }^{5}$ Vetsussie Faculty, University of Zürich and Radiotherapy, Hirslanden, Aarau, Switzerland. ${ }^{6}$ Department of Radiation Oncology, S. Camillo-Forlanini Hospital, Roma, Italy. ${ }^{7}$ Advanced Radiotherapy Center, European Institute of Oncology, Milano, Italy.

Received: 8 November 2012 Accepted: 20 January 2013

Published: 30 January 2013

\section{References}

1. Hodgson DC: Late effects in the era of modern therapy for Hodgkin lymphoma. Hematology Am Soc Hematol Educ Program 2011, 2011:323-329.

2. $\mathrm{Ng}$ AK: Review of the cardiac long-term effects of therapy for Hodgkin lymphoma. Br J Haematol 2011, 154(1):23-31.

3. Elkin EB, Klem ML, Gonzales AM, et al: Characteristics and outcomes of breast cancer in women with and without a history of radiation for Hodgkin's lymphoma: a multi-institutional, matched cohort study. J Clin Oncol 2011, 29(18):2466-2473.

4. Hoppe BS, Flampouri S, Lynch J, et al: Improving the therapeutic ratio in Hodgkin lymphoma through the use of proton therapy. Oncology (Williston Park) 2012, 26(5):456-459. 462-455.

5. Hoppe BS, Flampouri S, Su Z, et al: Effective Dose Reduction to Cardiac Structures Using Protons Compared with 3DCRT and IMRT in Mediastinal Hodgkin Lymphoma. Int J Radiat Oncol Biol Phys 2012, 84(2):449-455.

6. Li J, Dabaja B, Reed V, et al: Rationale for and preliminary results of proton beam therapy for mediastinal lymphoma. Int I Radiat Oncol Biol Phys 2011, 81(1):167-174.

7. Lu NN, Li YX, Wu RY, et al: Dosimetric and Clinical Outcomes of InvolvedField Intensity-Modulated Radiotherapy After Chemotherapy for EarlyStage Hodgkin's Lymphoma With Mediastinal Involvement. Int J Radiat Oncol Biol Phys 2012, 84(1):210-216.

8. Vlachaki MT, Kumar S: Helical tomotherapy in the radiotherapy treatment of Hodgkin's disease - a feasibility study. J App/ Clin Med Phys 2010, 11(1):3042.

9. Nieder C, Schill S, Kneschaurek P, et al: Comparison of three different mediastinal radiotherapy techniques in female patients: Impact on heart sparing and dose to the breasts. Radiother Oncol 2007, 82(3):301-307.

10. Fiandra C, Filippi AR, Catuzzo P, et al: Different IMRT solutions vs. 3DConformal Radiotherapy in early stage Hodgkin's lymphoma: dosimetric comparison and clinical considerations. Radiat Oncol 2012, 7(1):186.

11. De Sanctis V, Bolzan C, D'Arienzo M, et al: Intensity modulated radiotherapy in early stage Hodgkin lymphoma patients: is it better than three dimensional conformal radiotherapy? Radiat Oncol 2012, 7:129. 
12. Fox AM, Dosoretz AP, Mauch PM, et al: Predictive factors for radiation pneumonitis in hodgkin lymphoma patients receiving combinedmodality therapy. Int I Radiat Oncol Biol Phys 2012, 83(1):277-283.

13. Cella L, Conson M, Caterino M, et al: Thyroid V30 Predicts Radiation-Induced Hypothyroidism in Patients Treated With Sequential Chemo-Radiotherapy for Hodgkin's Lymphoma. Int J Radiat Oncol Biol Phys 2012, 82(5):1802-1808.

14. Cella L, Liuzzi R, Conson M, et al: Dosimetric predictors of asymptomatic heart valvular dysfunction following mediastinal irradiation for Hodgkin's lymphoma. Radiother Oncol 2011, 101(2):316-321.

15. Gagliardi G, Constine LS, Moiseenko V, et al: Radiation dose-volume effects in the heart. Int J Radiat Oncol Biol Phys 2010, 76(3 Suppl):S77-85.

16. Hall EJ, Wuu CS: Radiation-induced second cancers: the impact of 3D-CRT and IMRT. Int J Radiat Oncol Biol Phys 2003, 56(1):83-88.

17. Newhauser WD, Durante M: Assessing the risk of second malignancies after modern radiotherapy. Nat Rev Cancer 2011, 11(6):438-448.

18. Berrington De Gonzalez A, Gilbert E, Curtis R, et al: Second Solid Cancers After Radiation Therapy: A Systematic Review of the Epidemiologic Studies of the Radiation Dose-response Relationship. Int J Radiat Oncol Biol Phys 2012, Epub ahead of print.

19. Schneider U: Mechanistic model of radiation-induced cancer after fractionated radiotherapy using the linear-quadratic formula. Med Phys 2009, 36(4):1138-1143.

20. Schneider U, Sumila M, Robotka J: Site-specific dose-response relationships for cancer induction from the combined Japanese A-bomb and Hodgkin cohorts for doses relevant to radiotherapy. Theor Biol Med Model 2011, 8:27.

21. Schneider U, Sumila M, Robotka J, et al: Dose-response relationship for breast cancer induction at radiotherapy dose. Radiat Oncol 2011, 6:67.

22. Shuryak I, Hahnfeldt P, Hlatky L, et al: A new view of radiation-induced cancer: integrating short- and long-term processes. Part I: approach. Radiat Environ Biophys 2009, 48(3):263-274.

23. Shuryak I, Hahnfeldt P, Hlatky L, et al: A new view of radiation-induced cancer: integrating short- and long-term processes. Part II: second cancer risk estimation. Radiation and environmental biophysics 2009, 48 (3):275-286.

24. Cella L, Liuzzi R, Magliulo M, et al: Radiotherapy of large target volumes in Hodgkin's lymphoma: normal tissue sparing capability of forward IMRT versus conventional techniques. Radiat Oncol 2010, 5(1):33.

25. Feng $\mathrm{M}$, Moran $\mathrm{JM}$, Koelling $\mathrm{T}$, et al: Development and validation of a heart atlas to study cardiac exposure to radiation following treatment for breast cancer. Int J Radiat Oncol Biol Phys 2011, 79(1):10-18.

26. Weber DC, Peguret N, Dipasquale G, et al: Involved-node and involvedfield volumetric modulated arc vs. fixed beam intensity-modulated radiotherapy for female patients with early-stage supra-diaphragmatic Hodgkin lymphoma: a comparative planning study. Int J Radiat Oncol Biol Phys 2009, 75(5):1578-1586.

27. D'Errico F, Luszik-Bhadra M, Nath $R$, et al: Depth dose-equivalent and effective energies of photoneutrons generated by 6-18 MV X-ray beams for radiotherapy. Health Phys 2001, 80(1):4-11.

28. Schneider U, Agosteo S, Pedroni E, et al: Secondary neutron dose during proton therapy using spot scanning. Int J Radiat Oncol Biol Phys 2002, 53(1):244-251.

29. Engert A, Plutschow A, Eich HT, et al: Reduced Treatment Intensity in Patients with Early-Stage Hodgkin's Lymphoma. N Engl J Med 2010, 363(7):640-652.

30. Campbell BA, Hornby C, Cunninghame J, et al: Minimising critical organ irradiation in limited stage Hodgkin lymphoma: a dosimetric study of the benefit of involved node radiotherapy. Ann Oncol 2012, 23(5):1259-1266.

31. ICRP: Relative Biological Effectiveness, Radiation Weighting and Quality Factor". ICRP Publication 92. Ann. ICRP 33 (4); 2003.

32. Edwards AA, Lloyd DC, Prosser JS, et al: Chromosome aberrations induced in human lymphocytes by $8.7 \mathrm{MeV}$ protons and $23.5 \mathrm{MeV}$ helium-3 ions. Int J Radiat Biol Relat Stud Phys Chem Med 1986, 50(1):137-145.

33. NCRP: The relative biological effectiveness of radiations of different quality. NCRP Report 104; 1990.

34. Foote RL, Stafford SL, Petersen IA, et al: The clinical case for proton beam therapy. Radiat Oncol 2012, 7(1):174.

35. Schippers JM, Lomax AJ: Emerging technologies in proton therapy. Acta Oncol 2011, 50(6):838-850.

doi:10.1186/1748-717X-8-22

Cite this article as: Cella et al: Hodgkin's lymphoma emerging radiation treatment techniques: trade-offs between late radio-induced toxicities and secondary malignant neoplasms. Radiation Oncology 2013 8:22.

\section{Submit your next manuscript to BioMed Central and take full advantage of:}

- Convenient online submission

- Thorough peer review

- No space constraints or color figure charges

- Immediate publication on acceptance

- Inclusion in PubMed, CAS, Scopus and Google Scholar

- Research which is freely available for redistribution 